\title{
Eugène Green et la déclamation baroque : réinventer la langue?
}

Eugène Green and Baroque Declamation: Reinventing Language?

Julia Gros de Gasquet

\section{(2) OpenEdition}

Journals

Édition électronique

URL : https://journals.openedition.org/rsl/2459

DOI : $10.4000 /$ rsl.2459

ISSN : 2271-6246

Éditeur

Éditions Rue d'Ulm

Référence électronique

Julia Gros de Gasquet, « Eugène Green et la déclamation baroque : réinventer la langue ? ", Revue Sciences/Lettres [En ligne], 6 | 2019, mis en ligne le 10 décembre 2018, consulté le 30 juillet 2021. URL : http://journals.openedition.org/rsl/2459; DOI : https://doi.org/10.4000/rsl.2459

Ce document a été généré automatiquement le 30 juillet 2021

(C) Revue Sciences/Lettres 


\section{Eugène Green et la déclamation baroque : réinventer la langue?}

Eugène Green and Baroque Declamation: Reinventing Language?

Julia Gros de Gasquet

1 En France et dans le monde anglo-saxon, les années 1970 ont été le moment d'expériences sur la langue " ancienne ", celle des textes écrits aux XVII et XVIII siècles en Europe, qu'il s'agisse d'œuvres de fiction ou de traités savants. Deux pionniers ont joué un rôle décisif dans ce travail sur la langue, l'universitaire australien Dene Barnett ${ }^{1}$ et le metteur en scène, écrivain et réalisateur français Eugène Green, né à New York en 1947. En France, et en ce qui concerne la langue française, le travail d'Eugène Green constitue une expérience marquante. Il a en effet œuvré à retrouver, à restaurer, à reconstituer la langue dite baroque et avec elle, le corps de l'acteur baroque. L'hésitation sur les verbes rend compte de la difficulté à définir ce geste qui est une réactivation d'un état de la langue, à travers sa phonétique historique, son rythme et son intonation, mais aussi un geste esthétique qui invite à une pensée de l'acteur ancien. Pour le saisir, nous envisagerons ici tout d'abord le contexte de son émergence, pour en montrer la singularité. Mais avant cela, quelques éléments de biographie nous permettront de présenter brièvement Eugène Green :

Né en 1947 à New York, dans une famille originaire d'Europe centrale, il est arrivé en France en 1969, après être passé par Munich, Prague et Venise, la Vieille Europe où il lui a semblé retrouver sa propre mémoire. Désormais citoyen français, il a laissé derrière lui «le pays de la barbarie », entendez les États-Unis d'Amérique, et a fait, non pas tant de la langue de Molière que de celle de Racine, la langue dans laquelle il a d'abord écrit des pièces de théâtre, puis des essais, des romans, des scénarios et des dialogues. Il parle aussi l'allemand, l'italien et le portugais et vient de consacrer un documentaire de deux heures au basque qui l'intéresse en tant qu'il est une des langues les plus anciennes ${ }^{2}$.

On peut retracer son chemin en quelques dates : il crée en 1977 la compagnie théâtrale La Sapience. En 2001 son premier film, Toutes les nuits, reçoit le prix Delluc. En 2001 toujours, il publie La Parole baroque chez Desclée de Brouwer. En 2015, son film La Sapienza est distribué aux États-Unis. Le documentaire Faire la Parole sur la langue 
basque est diffusé en salle en 2017. En attendant les barbares, son dernier film, tourné en quelques jours à Toulouse, connaît un beau succès dans les différents festivals internationaux où il est montré en 2018. Aujourd'hui écrivain et cinéaste ${ }^{3}$, Eugène Green a initié un travail sur la langue dont il importe, pour le saisir, de le replacer dans son contexte :

J'ai été frappé par le décalage entre le traitement qu'on réservait au théâtre baroque, et l'évolution, que j'avais suivie avec passion et plaisir, de l'interprétation de la musique dite ancienne. Alors qu'auparavant ce répertoire s'était réduit à un sujet pour musicologues, parce qu'on disait - toujours en parlant à la première personne du pluriel - que notre oreille et notre sensibilité avaient évolué, rendant cette musique ennuyeuse et injouable, désormais, en cherchant à interpréter ces œuvres selon les techniques, et avec les instruments, de leur époque, Gustav Leonhardt et ses disciples leur donnaient une vie nouvelle. Alors je me suis posé la question : pourquoi ne pas en faire autant pour le théâtre ${ }^{4}$ ?

\section{Théâtre, danse et musique baroques}

3 Le contexte qui a nourri le travail d'Eugène Green est celui où s'épanouissent les propositions artistiques de danseurs, de musiciens et d'ensembles de musique qui ont adopté un style de jeu qui puise aux sources des traités anciens, de danse, de musique, et retrouve des instruments d'époque ${ }^{5}$.

4 Gustav Leonhardt (1928-2012) a initié à partir des années 1950 la redécouverte du répertoire de la musique ancienne, de ses sources, instruments et usages. Avec Nikolaus Harnoncourt (1929-2016), autre grand nom de la redécouverte de la musique ancienne, chef d'orchestre et gambiste, il enregistre entre 1971 et 1990 l'intégrale des cantates de Bach. Harnoncourt fonde le Concertus Musicus avec sa femme, Alice Hoffelner, en 1953. Cet ensemble se dédie à l'authentic performance («interprétation authentique ») sur instruments d'époque et acquiert dans les années 1970 une notoriété et une reconnaissance internationales. La discographie d'Harnoncourt marque ces années et fait entendre un son inouï, dans des interprétations contestées mais mémorables : les Concertos brandebourgeois (1964) et l'intégrale des cantates de Bach, L'Orfeo (1968) et L'Incoronazione di Poppea (1974) de Monteverdi, Il cimento delle'armonia e delle'inventione (1977) de Vivaldi, Belshazzar (1978), L'Ode à sainte Cécile (1978), Alexander's Feast (1979), Jephta (1979) de Haendel.

Harnoncourt a beaucoup écrit sur l'interprétation musicale ${ }^{6}$ et son travail a durablement marqué toute une génération d'artistes qui l'ont entendu, lu et qui ont étudié dans des départements de musique ancienne. Dans un même mouvement dont le théâtre n'est qu'un aspect, des artistes ont pensé la nécessité de revenir aux sources de la musique, de la danse et du théâtre des $\mathrm{XVII}^{\mathrm{e}}$ et $\mathrm{XvIII}$ e siècles en retrouvant les traces laissées par l'histoire dans des traités, dans des notations. Les danseurs, dont Francine Lancelot (1929-2003) en France fut la pionnière, ont retrouvé les traités anciens et les pas consignés par des notations anciennes, la notation Feuillet-Beauchamp par exemple. Ce commun mouvement est un acte de foi dans ces sources anciennes et une volonté partagée de revivifier, de réactiver un style d'interprétation musicale, de danse, de parole ou de chant. Le principe commun est celui d'une attention à l'histoire des pratiques anciennes et d'un retour aux sources pour retrouver des corps dansant, chantant et jouant. 
6 C'est dans le prolongement de ce mouvement que souhaite s'inscrire Eugène Green au moment où il crée sa compagnie de théâtre, La Sapience, en 1977 :

J'ai orienté mes études en suivant une attirance, ressentie depuis mon adolescence, pour la Renaissance et l'époque baroque. Je me suis aperçu que cette conception du théâtre que je trouvais si bouleversante, et si familière, dans les théâtres d'Asie, avait également existé en Europe pendant ces grands siècles de création artistique. D'autre part, le renouveau de l'interprétation de la musique de ces périodes, à la fin des années 70, a fourni l'exemple d'un héritage culturel actualisé par un effort de retrouver un passé au présent. Alors j'ai formé un projet analogue pour le théâtre baroque. La nécessité de cette démarche me semblait évidente, aussi bien pour l'évolution de la culture théâtrale contemporaine que par rapport à mon chemin personnel. Je pensais que cette proposition s'insérerait dans le paysage théâtral avec pas plus de heurts, et de manière aussi inéluctable, que je voyais se faire accepter les nouvelles interprétations musicales. Constatant qu'aucune recherche n'existait qui me permettrait de passer directement à une pratique artistique, j'ai commencé à faire ce travail moi-même. Je l'ai fait avec tout le sérieux dont j'étais capable, et j'ai eu la chance de pouvoir suivre, à l'école des Hautes études en sciences sociales, le séminaire de Louis Marin; au cours de mes études et de mon travail, j'ai pu également profiter des recherches et de l'amitié de plusieurs grands spécialistes de la période qui m'intéressait. Néanmoins, contrairement aux universitaires, mon but n'a jamais été de faire apparaître une vérité historique : ma recherche n'a de valeur que par rapport à un chemin artistique et spirituel ${ }^{7}$.

\section{Corps et voix de l'acteur baroque}

7 Le travail dont Eugène Green a été le pionnier en France est bien celui de la redécouverte du baroque, ce terme venant qualifier tant la déclamation dont il a recherché les sources que les acteurs et actrices formés et formées à ce style de jeu. L'adjectif "baroque » concentre, en un feuilleté sémantique complexe, des acceptions qui se chevauchent, se complètent et s'enrichissent. La question de la définition du mot est vaste et la mise au point de Fabien Cavaillé ${ }^{8}$ permet de réfléchir à partir de deux niveaux de sens. "Baroque" renvoie à un répertoire et plus généralement à une production littéraire et artistique européenne qui a cours du $\mathrm{XVI}^{\mathrm{e}}$ au $\mathrm{XVIII}^{\mathrm{e}}$ siècle, mais aussi à une manière de "performer " ce répertoire, qu'il s'agisse de danse, de déclamation ou d'opéra :

Parmi toutes ces extensions sémantiques du baroque, comment situer le glissement de sens qui s'est imposé dans le langage artistique et scientifique, à partir des années 70-80 ? En effet, on s'est mis à parler de musique baroque ou de théâtre baroque pour dire à la fois le répertoire et une manière de le jouer, en se fondant sur les règles et les usages de l'interprétation musicale ou vocale à l'époque dite baroque. Ainsi, d'une même œuvre baroque, on pourra avoir aussi bien une interprétation actuelle moderne qu'une interprétation actuelle baroque puisque fondée sur des codes historiquement justes. Toute la question est de savoir si baroque, dans mise en scène baroque, a encore les sens que l'on évoque traditionnellement pour les œuvres ou si le terme est seulement là pour signifier une certaine méthode, un "parti pris » ou un geste artistique: dit-on baroque parce que le nom est plus élégant qu'historiquement fondé ou archéologique? [...] En ce sens, les spectacles baroques, comme ceux de l'Antiquité, comme toutes les formes anciennes et oubliées, peuvent apparaître aux artistes comme un nouvel horizon, un chemin qui, tout en faisant un retour en arrière, amène aux questions et aux formes de notre temps 9 . 
8 Ainsi entendu, le «baroque » est une mise en mouvement, qui projette l'Histoire dans le présent sous la forme de spectacles, de questionnements théoriques et pratiques que partagent les chercheurs, les praticiens et les artistes.

9 Les recherches d'Eugène Green ont commencé par un travail sur l'adéquation entre le lieu de la parole et la rhétorique. Dans "Le "lieu" de la déclamation tragique ${ }^{10}$ ", Eugène Green partait de l'idée des trois lieux de l'éloquence au XVII ${ }^{\mathrm{e}}$ siècle : le théâtre, la chaire, le barreau, auxquels peut se rajouter un quatrième lieu, celui du salon savant. Ces lieux de la parole publique réclament un mode de diction qu'ils ont en partage et que l'on nomme déclamation. À partir du constat d'une communauté de principe dans l'éloquence de la chaire, du théâtre et du barreau, Eugène Green prolongeait l'analyse en apportant une idée essentielle pour le devenir de la pratique théâtrale des textes du $\mathrm{XVII}^{\mathrm{e}}$ siècle : la nécessité de dire avec un ton de voix emphatique toute parole prononcée dans un des lieux de l'éloquence. Et ce faisant, il abordait plus largement le travail de l'acteur et son rapport à la rhétorique :

Toute théorie baroque de la représentation théâtrale part du principe que le jeu scénique est une application de l'actio de la rhétorique [...]. Les écrits théoriques latins sur la rhétorique, essentiellement De oratore et De inventione de Cicéron, le Ad Herennium qui lui était attribué, et De Institutione oratoria de Quintilien, ont été analysés et réinterprétés par rapport à la pratique moderne dans le grand ouvrage du jésuite Louis de Cressolles, Vacationes autumnales, publié à Paris en $1620^{11}$

En partant de cette source essentielle que représentent les Vacationes ${ }^{12}$ et en s'appuyant par ailleurs sur les traités français de Michel Le Faucheur (Traité de l'action de l'orateur, ou de la prononciation et du geste, Paris, 1676) et René Bary (Méthode pour bien prononcer un discours et pour le bien animer, Paris, 1679), sur le traité anglais de John Bulwer (Chirologia et Chironomia, Londres, 1644) et le traité italien de Giovanni Bonifacio, L'Arte de'cenni (Vicence, 1614, deuxième édition 1616), Eugène Green a souhaité mettre au point une théorie de la représentation gestuelle :

L'homme baroque estimait que le geste était un langage naturel et universel, qui, sous sa forme primitive, et entièrement muette, permettait aux êtres humains de communiquer des idées et des sentiments. [...] L'absence totale de références à un «trésor » de vocabulaire gestuel indique clairement que s'il y avait des règles et des principes généraux pour l'action oratoire, ainsi qu'un fond commun de gestes, il n'y avait pas en revanche de codification aussi précise que celle, par exemple, du Kathakali. L'orateur était libre d'inventer ses gestes, de faire des choix, comme pour la déclamation, et l'acteur pouvait modifier des éléments de sa gestuelle d'une représentation à une autre ${ }^{13}$.

11 Les principes de rhétorique concernant l'action de l'orateur, et qui informent le jeu de l'acteur, s'appuient sur l'expression des passions : comment exprimer vocalement et gestuellement telle ou telle passion que le poète aura donnée à son personnage ? Les passions sont liées à la théorie médicale $d u X \mathrm{XII}^{\mathrm{e}}$ siècle qui nous apparaît aujourd'hui comme fantasque, mais qui avait alors le mérite de mettre en relation le corps et les affections de l'âme, et d'expliquer par exemple pourquoi le corps d'une femme amoureuse pouvait « et transir et brûler » selon les mots de Racine ${ }^{14}$. Les passions sont des affections de l'âme matérialisées par des symptômes corporels :

D'où l'on peut conclure que les anciens ont établi ces quatre passions, à savoir la joie, la douleur, la crainte et l'espérance, comme les quatre éléments, ou les quatre humeurs de l'appétit qui nous est commun avec les animaux. [...] Or il faut voir en quoi consiste le mouvement de ces passions avant que de leur établir de certains accents. En premier lieu, le cœur s'élargit, s'épanouit et s'ouvre dans la joie et dans 
l'espérance, comme l'héliotrope, la rose et le lis à la présence du soleil. De là vient que le teint du visage est vermeil à cause des esprits vitaux que le cœur envoie en haut. [...] La joie amène quantité de sang et d'esprit au visage, et aux autres parties $\mathrm{du}$ corps. Mais la crainte et la douleur sont semblables au reflux qui retire ce qu'il avait amené : car la crainte et l'effroi rendent le visage pâle et la contenance morne et hideuse ${ }^{15}$.

12 Les traités à l'usage de l'orateur tels qu'ils s'écrivent au XVII ${ }^{\mathrm{e}}$ siècle suivent toujours le même plan, en deux parties : la première consacrée à la voix, la deuxième consacrée au corps de l'orateur. C'est la même codification qui prévaut pour régler voix et corps. Les derniers chapitres du traité de Michel Le Faucheur cité plus haut sont consacrés au geste :

Qu'il [le geste] paraisse naturel et né des choses que vous dites et de l'affection qui vous meut à les dire. [...] Pour le corps entier, il ne doit changer ni de place, ni de posture à tout moment. Mais il ne faut pas aussi qu'il demeure immobile comme un tronc. [...] Il faut faire tous les gestes de la main droite. Si vous haussez la main, ce ne doit pas être plus haut que les yeux. Il faut que vos yeux voient toujours vos mains, qu'elles environnent toujours votre tête, vous ne devez guère étendre vos bras plus loin d'un demi-pied du tronc de votre corps, les gestes doivent être fort médiocres et fort modestes ${ }^{16}$.

13 On voit comment la gestuelle de l'orateur est encadrée par des règles précises, de port des bras, des mains, d'accompagnement du texte par un certain nombre de gestes.

Les gestes restent «médiocres et modestes » chez Le Faucheur. De même, l'emphase vocale est de l'ordre du supplément d'énergie, non pas du grossissement. Tout concourt à faire de cet art de l'éloquence un art extrêmement codifié par les règles et canons rhétoriques mais cet art doit se dissimuler, c'est ici qu'intervient la feinte. Ce que vient saluer le public au théâtre, c'est l'art de la dissimulation. Le naturel est le résultat de cette dissimulation. Charles Sorel définit très bien cet art de la feinte :

Le plus grand Art qu'il y ait, c'est de bien savoir cacher l'Art : c'est par là qu'on ravit les hommes en admiration, leur faisant croire que l'on parle bien naturellement et sans affectation aucune. L'artifice même étant réitéré, nous donne enfin une accoutumance à bien dire ${ }^{17}$.

15 Les bienséances interviennent ici aussi dans la mesure où certains gestes ne peuvent convenir à l'orateur :

[...] Le mouvement des mains doit convenir à la nature des actions dont on parle [...]. Il y a particulièrement des actions que vous ne devez jamais essayer de représenter avec les mains, ni vous mettre en la posture de ceux qui les font, comme d'escrimer, de bander un arc, de tirer un coup de mousquet, [...] les gestes doivent être fort médiocres et fort modestes, et non point grands et vastes, ni aussi trop fréquents, ce qui serait une agitation de bras et de mains messéante à un orateur $[\ldots]^{18}$.

16 Dans la même veine, le traité de rhétorique de René Bary indique comment les gestes doivent varier en fonction des passions en jeu ou des figures de rhétorique exprimées dans le texte :

Le triomphe veut qu'on regarde le Ciel, comme de côté, qu'on porte le bras droit vers le bras gauche, et que l'on baisse un peu la tête, parce que le triomphe suppose qu'on emporte tout de grand, et que cette action marque comme un progrès momentané [...].

L'étonnement, au moins celui qui naît des choses fâcheuses veut qu'on envisage l'auditoire d'un œil extraordinairement ouvert, qu'on remue lentement la tête de côté et d'autre, et qu'en écartant les bras tombant, on ouvre les mains, parce que l'étonnement qui naît des choses fâcheuses, est une surprise comme glaçante; et 
que dans une surprise de cette nature, l'âme n'étant presque que dans les yeux, semble avoir abandonné les autres parties.

[...] L'ironie veut qu'on tourne la tête du côté gauche, et qu'on parle d'un ton exclamatif et railleur.

[...] La colère, ou pour mieux dire la colère présomptueuse, veut qu'on élève horriblement les paupières, et qu'on avance même la lèvre inférieure [...] parce que dans la vengeance l'œil enflammé et la lèvre inférieure avancée marquent l'animosité ${ }^{19}$.

L'acteur est ainsi vocalement et gestuellement amené à une maîtrise qui signe son savoir-faire et témoigne de la virtuosité de son art.

\section{La langue baroque est une parole : rythme, hauteur et phonétique historique}

18 Cette présentation des codes qui régissent le travail de l'acteur ancien ne serait pas complète sans un complément plus « technique » et plus concret sur la manière dont la langue baroque se fabrique en termes de phonétique (les sonorités), de rythme et d'accentuation. Elle s'articule en effet autour de ces trois points qui, ensemble, la constituent et signent sa singularité. Nous en donnerons ici les éléments essentiels.

Le phénomène naturel d'évolution de la langue française veut que certaines graphies ne se prononcent plus aujourd'hui comme les locuteurs en avaient l'habitude il y a quatre siècles. Au XVII ${ }^{\mathrm{e}}$ siècle :

le -ll- géminé était mouillé,

le -h-était aspiré,

le -r- était roulé,

les voyelles nasales ein, ain, in, et an et en, à l'exception de un et on étaient prononcées en deux temps [é-n], ou [a-n],

le -e- dit muet se prononçait,

la graphie oi se prononçait [wé],

les consonnes finales des vers ou à la pause, en prose comme en vers, se faisaient entendre ${ }^{20}$.

Ce qui étonne le plus le public - et peut le rebuter ou au contraire l'envoûter - est, d'une part, la bizarrerie des sons de la langue qui sonnent comme étrangers à nos propres oreilles, et, d'autre part, « la musique » présente dans ces textes.

21 Car à cette phonétique historique s'ajoute le travail de déclamation qui consiste à porter la langue avec énergie, selon un rythme que se donne l'interprète, et en travaillant des accents de la langue en hauteur (intonation) et en intensité (accents rhétoriques). Cela concourt à donner à la voix une direction dans l'espace qui épouse, en fonction de l'écriture du texte, des montées et des redescentes, façonnant un schéma harmonique. La musique de la langue «baroque » ouvre ainsi dans les années 1970 un débat qui n'est pas nouveau. Paul Valéry en faisait déjà état dans les années 1920. Invité à donner des conseils à de jeunes comédiens qui, en 1925, montaient Bajazet à la Petite Scène, il leur adressa l'année suivante un discours où il résumait et synthétisait sa conception de la diction du vers classique et notamment de l'alexandrin racinien. Ce qui doit primer, selon Paul Valéry, dans l'exécution d'un texte versifié, c'est la musique :

Le poème comme un morceau de musique n'offre en soi qu'un texte qui n'est rigoureusement qu'une sorte de recette. [...] Le poème est une abstraction, une 
écriture qui attend, une loi qui ne vit que sur quelque bouche humaine et cette bouche est ce qu'elle est ${ }^{21}$.

Valéry se situe résolument du côté de ceux qui pensent que le vers doit être chanté pour être entendu. Le langage courant ne peut donc être la référence de l'acteur qui dit l'alexandrin classique :

[...] il ne faut point, dans l'étude d'une pièce de poésie que l'on veut faire entendre, prendre pour origine ou point de départ de sa recherche le discours ordinaire et la parole courante pour s'élever de cette prose plane jusqu'au ton poétique voulu; mais, au contraire, je pensais qu'il faudrait se fonder sur le chant, se mettre dans l'état du chanteur, accommoder sa voix à la plénitude du son musical, et de là redescendre jusqu'à l'état un peu moins vibrant qui convient aux vers. [...] Avant toute chose, bien poser la voix fort loin de la prose, étudier le texte sous le rapport des attaques, des modulations, des termes qu'il comporte, et réduire peu à peu cette disposition qu'on aura exagérée jusqu'aux proportions de la poésie ${ }^{22}$.

La voix participe de la musique et du sens. Or, pour faire parvenir le sens à l'auditeur, il faut respecter la musique. C'est grâce à la plénitude de la musicalité des vers qu'on peut accéder au sens.

La première condition pour bien dire les vers est d'avoir compris ce qu'ils ne sont pas, et quelle immense différence les sépare du langage ordinaire. La parole plane et courante, celle qui sert à quelque chose, vole à sa signification, à sa traduction purement mentale, et s'y abolit, et s'y fond, comme un germe dans l'œuf qu'il féconde. [...] Mais le vers a pour fin une volupté suivie, et il exige, sous peine de se réduire à un discours bizarrement et inutilement mesuré, une certaine union très intime de la réalité physique du son et des excitations virtuelles du sens. [...] le chant, d'une part, et la prose de l'autre, sont placés comme symétriquement par rapport au vers, lequel s'établit dans un équilibre admirable et fort délicat entre la force sensuelle et la force intellectuelle du langage $e^{23}$.

Les détracteurs de la déclamation baroque ${ }^{24}$ l'accusent de n'être que mélopée musicale qui éloigne du sens, qui fait perdre le discours aux auditeurs-spectateurs. Les défenseurs de la déclamation baroque, car il s'agit bien d'une guerre polémique qui s'est ourdie autour d'elle, répondent au contraire que la déclamation, en faisant entendre les accents rhétoriques du texte, amène les auditeurs-spectateurs à une compréhension qui passe par la construction des périodes et le souffle de ceux qui les disent. Mais la musique peut devenir un autre point d'accroche pour ajouter un argument dans la polémique : "À la différence de la musique, où existent encore les instruments d'époque et les partitions, il n'y a aucune manière de retrouver les voix d'acteurs, leurs dictions ${ }^{25}$.» Les musiciens seraient ainsi plus autorisés que les comédiens dans ce travail de réactivation historique car les instruments baroques peuvent être retrouvés, et les partitions sorties des bibliothèques, intactes comme au jour de leur exécution. Or, cette assertion de François Regnault est clairement démentie par le travail de musiciens spécialistes de musique ancienne qui démontrent combien les interprètes en musique, tout comme les comédiennes et comédiens, sont placés devant des lacunes, des traces difficiles à interpréter qui leur demandent d'être créatifs et non pas « exécutants » d'une musique dont toute la mémoire d'interprétation serait clairement identifiable et identifiée ${ }^{26}$.

Depuis quelques années, à cette polémique menée par des détracteurs extérieurs à la déclamation baroque, s'est ajoutée une seconde polémique, initiée par des spécialistes qui reviennent sur certaines propositions d'Eugène Green quant à la prononciation de certaines lettres ou syllabes (consonnes finales des mots, infinitifs en er) ${ }^{27}$. 
Depuis 2001 - bien avant cette discussion de spécialistes -, Eugène Green, qui a abandonné le théâtre, se consacre à sa vie d'écrivain et de cinéaste. Il ne parle pas de la déclamation baroque en termes d'héritage, mais plutôt comme d'une forme dont ses élèves se sont nourris pour mener à bien leur propre chemin dans la langue et dans le théâtre :

Depuis ma retraite effective du théâtre - mais non de l'activité artistique - il y a seize ans, le théâtre baroque continue. Des comédiens-metteurs en scène comme Benjamin Lazar et Louise Moaty, sortis des ateliers du lycée Montaigne, ou des comédiens avec qui j'avais travaillé, comme Anne-Guersande Ledoux, Manuel Weber, Julia de Gasquet, Sophie Delage, et Alexandra Rübner, continuent à créer des spectacles et à enseigner des techniques dérivées de ma proposition initiale. Il va de soi que leur travail reflète leurs propres interprétations et le sens personnel qu'ils y trouvent, ce qui n'est que trop souhaitable. Mais l'idée reste vivante. [...] En 2001 mon premier film, tourné deux ans plus tôt, est sorti en salle, et cette même année, bien que j'écrive quotidiennement depuis mon adolescence, j'ai vu mon premier livre publié. Depuis je trouve une grande satisfaction à réaliser des films et à écrire des livres qui sont édités. Dans l'un et l'autre cas leur public demeure restreint, mais il est infiniment plus vaste que celui que j'ai connu en faisant du théâtre, et traverse les frontières.

D'un point de vue social, mon modeste projet de faire revivre le théâtre baroque a été un échec, mais je ne regrette nullement de l'avoir proposé, car sa lumière me nourrit dans mon existence humaine, et dans le travail artistique que je fais actuellement. J'espère aussi que cette tentative éclaire un peu le chemin de ceux qui en ont été témoins, ou qui en ont hérité la pratique, sous une forme ou une autre. C'était en tout cas, pour moi, une chose absolument nécessaire, et comme on le voit dans toutes les grandes tragédies, l'anangké, la nécessité, est toujours douloureuse, mais aboutit à la sérénité, et à la joie ${ }^{28}$.

La lucidité apaisée d'Eugène Green ouvre un chemin plein de sérénité pour sortir des polémiques vaines, et nous aide à poser les seules questions véritablement pleines d'enjeux concernant la déclamation baroque aujourd'hui : comment le public la reçoitil ? Pourquoi des comédiennes et comédiens s'y adonnent-ils? Que l'on doive prononcer plus ou moins de consonnes latentes est sans doute un sujet d'interrogation important, d'une part, pour les spécialistes, d'autre part, pour les comédiens qui souhaitent être "historiquement informés ", mais aux oreilles d'un public de théâtre contemporain, cela ne fera pas grande différence. Il entendra, d'une manière ou d'une autre, l'étrangeté de cette langue dans sa propre langue, et réagira à cette étrangeté.

Le public des spectacles baroques est souvent envoûté, étonné après une représentation ${ }^{29}$. Certains spectateurs, heurtés, préfèrent partir. La réception a toujours été ainsi fortement marquée par l'enthousiasme ou le net rejet. Pour les comédiens et comédiennes qui pratiquent cet art, le travail gestuel comme celui des amplitudes vocales sont autant de paramètres «artificiels » avec lesquels construire leur jeu. Cela leur permet de ne pas jouer "psychologiquement»; ils peuvent convoquer non seulement des sensations et des émotions personnelles mais s'amuser à les élargir, les agrandir, les amplifier par le travail des intonations musicales, par la puissance du geste, et trouver du même coup une sincérité qui ne soit pas naturaliste. La déclamation baroque demeure donc, près de quarante ans après sa "réinvention ", une langue qui divise, une langue que ne parle pas toute la profession théâtrale, une langue souvent plébiscitée par le public, dans sa bizarrerie et sa beauté. 
Jean Racine, Andromaque, Acte III scène 8, v. 1001-1015, voix et enregistrement Julia Gros de Gasquet.

docannexe/file/2541/gros_andromaque.mp4

\section{NOTES}

1. On retiendra notamment The Art of Gesture: The Practices and Principles of 18th Century Acting, Heidelberg, Carl Winter Universitätsverlag, 1987.

2. Catherine Millet, «Eugène Green, un parfait Français », http://next.liberation.fr/cinema/ 2015/03/18/eugene-green-un-parfait-francais_1223356, consulté le 12 mars 2018.

3. Parmi ses derniers films, Le Fils de Joseph, sorti en 2016. Parmi ses derniers livres, Les Voix de la nuit, Paris, Robert Laffont, 2017, et L'Enfant de Prague, Paris, Phébus, 2017.

4. « Entretien avec Eugène Green ", in Scènes baroques d'aujourd'hui. La mise en scène baroque dans le paysage culturel contemporain, Céline Candiard et Julia Gros de Gasquet (dir.), Lyon, Presses universitaires de Lyon, à paraître en 2019.

5. Théorbe, luth, viole de gambe, pour n'en citer que quelques-uns, accordés au la 415, soit un demi-ton en-dessous du diapason communément admis, le la 3, $440 \mathrm{~Hz}$ (soit 440 vibrations/ seconde)

6. On peut citer Le Discours musical. Pour une nouvelle conception de la musique [Musik als Klangrede: Wege zu einem neuen Musikverständnis] (trad. de l'allemand par Dennis Collins), Paris, Gallimard, «Tel », 2014, et Le Dialogue musical. Monteverdi, Bach et Mozart [Der musikalische Dialog : Gedanken zu Monteverdi, Bach und Mozart] (trad. de l'allemand par Dennis Collins), Paris, Gallimard, « Arcades » $\left(\mathrm{n}^{\circ} 7\right), 1985$.

7. Eugène Green, La Parole baroque, Paris, Desclée de Brouwer, 2001, p. 13-14.

8. Fabien Cavaillé, "Qu'avez-vous dit avec "baroque”? Extension d'une notion, usages d'une étiquette", in Scènes baroques d'aujourd'hui. La mise en scène baroque dans le paysage culturel contemporain, op. cit.

9. Ibid.

10. Eugène Green, «Le "lieu" de la déclamation tragique ", Littératures classiques, n 12, 1990, p. 275-291.

11. Eugène Green, La Parole baroque, op. cit., p. 136.

12. Ibid.

13. Ibid., p. 144-145.

14. Voir Jackie Pigeaud, La Maladie de l'âme, Paris, Les Belles Lettres, 1989.

15. Père Marin Mersenne, L'Harmonie universelle contenant la théorie et la pratique de la musique, Paris, Sébastien Cramoisy, 1636, "Livre de l'art de bien chanter », proposition XII, p. 20 et sq.

16. Michel Le Faucheur, Traité de l'action de l'orateur, ou de la prononciation et du geste, Paris, Conrart, 1676, chapitres XII et XIII, p. 225 et sq.

17. Charles Sorel, De la manière de bien parler, chapitre I, in De la connaissance des bons livres, Paris, André Pralard, 1671, réimpression Slatkine, Genève-Paris, 1981, p. 270.

18. Le Faucheur, op. cit., p. 220, 224, 225, 226.

19. René Bary, Méthode pour bien prononcer un discours et pour le bien animer, Paris, chez Denys Thierry, 1679, p. 82 à 93. (Nous soulignons.)

20. Voir Eugène Green, La Parole baroque, op. cit., p. 281-285. 
21. Paul Valéry, Discours de la diction des vers, prononcé par M. Paul Valéry de l'Académie française, au dîner annuel de la Revue Critique, le 27 mai 1926, Paris, Société « Le Livre », 1926, p. 15-16.

22. Ibid., p. 16-17. (Nous soulignons.)

23. Ibid., p. 18-20.

24. Parmi eux, on peut citer Marc Fumaroli. Voir l'article de Nicolas d'Estienne d'Orves, «Comment parlaient les personnages de Racine?», paru dans Le Figaro du 21 octobre 1999, ou encore Jean-Marie Villégier dans un article paru dans Télérama en mai 2011: http:// www.telerama.fr/scenes/jean-marie-villegier-metteur-en-scene-du-grand-siecle,68494.php (consulté le 28 avril 2018).

25. François Regnault, cité par Mathilde La Bardonnie dans un article paru dans Libération le 28 septembre 1999, « Racine en vers et contre tous. Eugène Green propose une version baroque de Mithridate ».

26. Voir l'article du musicien Benjamin Narvey, «Le modèle musical baroque aujourd'hui, entre attention aux sources et créativité ", in Scènes baroques contemporaines, op. cit.

27. Sur cette polémique, voir le récit de Bénédicte Louvat-Molozay, « Recherche universitaire et pratique théâtrale : un dialogue impossible? ", ibid.

28. Eugène Green, « La brève histoire d'une proposition modeste ", ibid.

29. Au Festival de la Correspondance de Grignan en juillet 2017, lors de la lecture baroque d'un choix de lettres de Madame de Sévigné à sa fille, Madame de Grignan, la discussion avec un public non averti, peu ou pas familier des plateaux baroques, a fait surgir très vite la projection vers l'œuvre, plutôt que la translation de l'œuvre ancienne dans l'époque actuelle, comme l'enjeu majeur de cette forme d'art. Voir le programme en ligne: https://www.grignanfestivalcorrespondance.com/img/pdf/Programme-2017.pdf (consulté le 12 août 2017). La lecture était ainsi présentée aux spectateurs : «Comment nous rendre contemporains de Madame de Sévigné et pourquoi s'y risquer ? À partir d'un choix de lettres courant sur une année épistolaire (novembre 1670-novembre 1671), un travail de réactivation baroque qui s'appuie sur le chant, la déclamation et la gestuelle $d u x_{\text {II }}{ }^{\mathrm{e}}$ siècle sera montré.» Le public s'est montré curieux et enthousiaste lors de cette lecture par Louise Moaty, Julia de Gasquet et Jeanne Zaepffel, et au cours de la discussion qui a suivi.

\section{RÉSUMÉS}

Cet article présente le travail théâtral du cinéaste et écrivain qu'est aujourd'hui Eugène Green. À travers son rapport à la langue française se fait jour la proposition à la fois théorique et artistique qu'il a initiée dans les années 1970 en France. Cette proposition s'appuie sur la déclamation qui respecte un état phonétique historique de la langue, et considère que la parole éloquente est d'abord un geste. Ce geste est saisi dans le contexte historique qui le voit naître et l'on suivra le développement de cette proposition sur l'arc temporel qui va de 1977, date à laquelle Eugène Green fonde Le Théâtre de la Sapience, jusqu'en 2001, date à laquelle il renonce à son activité théâtrale. À travers ce récit de vie et d'expérience, affleure la raison d'être foncière de la pratique artistique d'Eugène Green, en termes de poétique et de réception : quel acteur façonne-t-elle ? À quel public s'adresse-t-elle?

The article focuses on the dramatic work of filmmaker and writer Eugène Green. In the 1970s in France, Green initiated a theoretical and artistic proposition that stems from his relationship to 
the French language. This proposition is rooted in the art of declamation that respects a historical phonetic state of language and views eloquent speech as a gesture above all, one that is understood within the historical context that produces it. We follow the development of this proposition, which spanned the period from 1977, when Eugène Green founded Le Théâtre de la Sapience, until 2001, when his theatre activity came to an end. This account of his life and experience touches on the essential purpose of Eugène Green's artistic practice in terms of poetics and reception: What actor does it shape? For what audience is it intended?

\section{INDEX}

Keywords : baroque, declamation, rhythm, intonation, rhetoric, expression of passions, gesture, technique

Mots-clés : baroque, déclamation, rythme, intonation, rhétorique, expression des passions, geste, technique

\section{AUTEUR}

\section{JULIA GROS DE GASQUET}

Universitaire et comédienne, Julia Gros de Gasquet est maître de conférences HDR à l'Institut d'études théâtrales de l'université Sorbonne Nouvelle-Paris 3. Comme comédienne, elle s'est formée à l'ENSATT à Lyon et au GITIS à Moscou. Au cinéma, elle a été dirigée par Eugène Green dans Le Pont des Arts et dans Le Fils de Joseph (2016). Spécialiste du jeu de l'acteur, elle a écrit à plusieurs reprises sur ce sujet et publie une synthèse de ses travaux pour l'HDR dans la livraison de janvier 2019 de la Revue d'histoire du théâtre sous le titre « Pour une histoire de l'art de l'acteur : perspectives et propositions ». Elle est la directrice artistique du Festival de la correspondance de Grignan depuis 2015.

Parmi ses publications :

En disant l'alexandrin. L'acteur tragique et son art, $17^{e}$ siècle-20 siècle, Paris, Champion, 2006.

La Voix du public. Manifestations sonores des spectateurs et spectatrices aux $17^{e}$ et $18^{e}$ siècles, J. Gros de Gasquet et S. Nancy (dir.), Rennes, PUR, à paraître en 2019.

L'Objet technique en scène, J. Gros de Gasquet et J. Valero (dir.), Montpellier, Deuxième Époque, à paraître en 2019.

Scènes baroques contemporaines, J. Gros de Gasquet et C. Candiard (dir.), Lyon, PUL, à paraître en 2019. 